\title{
Multilayer Convolutional Neural Network for Plant Diseases Detection
}

\author{
Divya Verma, Gurpreet singh, Hatesh Shyan
}

\begin{abstract}
Plant diseases are causing a significant loss in the agriculture production. The diseases infect the plant leaves, stem and the fruits which cannot be utilized. The main causes of plant diseases are bacteria, fungi and virus. The identification and diagnosis of the diseases are necessary. Many researchers have delved deeper in this field and find suitable techniques to this end. Moreover, these days, Convolution Neural network has attracted the interest of the researchers as it gives better results for image processing. This paper presents a comparative analysis of the various approaches designed to diagnose the diseases in different plant at the initial stage so that preventive measure can be taken to enhance the productivity. Along with this, the role of $C N N$ in detecting the disease in the plants is also described in this paper.
\end{abstract}

Keywords: CNN, plant disease detection, neural network, feature extraction

\section{INTRODUCTION}

Agriculture industry plays necessary function in preserving the economic system of countries. One of the fundamental reasons of monetary loss experienced on the international degree in this quarter is the illnesses precipitated to the plants. There are a variety of signs proven by using the flora from the initial to the final segment of the disease. The music of the modifications can be recorded with the help of environment friendly technological know-how in surveillance systems. This is a very challenging task. While the sight and appreciation of people is astonishingly influential in recognizing and appreciation special patterns. Analyzing the ailment visually, The visible assessment of plant ailments is a subjective project which is in addition is primarily based on psychological phenomenon that might also result in biased, optical illusions and, eventually, to error. [1] Globally, yielding and the quality of the agriculture production get affected by the plant diseases and these diseases are also responsible for the noteworthy portion of costs spent on production. According to a survey, more than $10 \%$ of the food production is decreased due to the plant diseases [2].

Manuscript published on June 30, 2020.

* Correspondence Author

Divya Verma*, ReSearch Scholar, Department of Computer Science Engineering, Chandigarh University, Punjab, India

Gurpreet Singh, Assistant Professor, Chandigarh University, Punjab

Hatesh Shyan. Assistant Professor, Department of Computer Science \& Engineering, Chandigarh University, Pujab, India.

(C) The Authors. Published by Blue Eyes Intelligence Engineering and Sciences Publication (BEIESP). This is an open access article under the CC BY-NC-ND license (http://creativecommons.org/licenses/by-nc-nd/4.0/)
However, the advancement in the technology in terms of machine learning and computer vision, a great progress is made in the field of identifying and diagnosing of plant diseases [3][4]. Basically, diseases causes harm to the normal states of the plants which in turn alters the important functions such as transpiration, photosynthesis, fertilization, pollination, germination and so on. The main causes of these diseases are pathogens such as bacteria, viruses and fungi. Adverse conditions in environment can be another reason of unhealthy plants. Thus, it is important to detect the disease at its initial stage. [5]. For instance, ailments in pomegranate plant can be located in exclusive components such as leaves, fruits, and stem. The ailments that affect the fruit are anthracnose (Colletotrichum gloeosporoides), bacterial blight (Xanthomonas axonopodis pv punicae), and wilt complex (ceratocystis fimbriata). Also, disease located in the mango leaves is Anthracnose which is causes due to fungus. These illnesses are detected with the assist of computer visions and are further treated in order to acquire the fine food. Moreover, deep mastering is the most outstanding technology to recognize the plant diseases. It is referred to as the use of artificial neural community (ANN) structure in which a quantity of processing layers are present. Now, the models of deep mastering has been reached to the area of photo recognition which help in the agriculture industry to maintain their productiveness by using retaining the plant away from diseases or treating them at the early stage of ailments [6][7]. Generally, the indispensable tool utilized for deep mastering in this region is Convolutional Neural Networks (CNNs) [6]. CNN is one of the influential mechanisms that models the complex processes and also performs sample consciousness in the purposes carrying massive quantity of statistics such as images. CNN is used with the aid of a variety of researchers to discover and diagnose the diseases in real life conditions.

In this paper, an evaluation of a range of techniques and mechanism opted by many researchers is presented. The paper is divided into four sections which describe the types of diseases, Convolution neural network, and the literature survey respectively.

\section{TYPES OF DISEASES}

There are various germs that cause the plant diseases shown in figure 1 . These are broadly classified into three types. In this section, the symptoms of the diseases in the plant due to different germs such as bacteria, fungi and virus are explained.

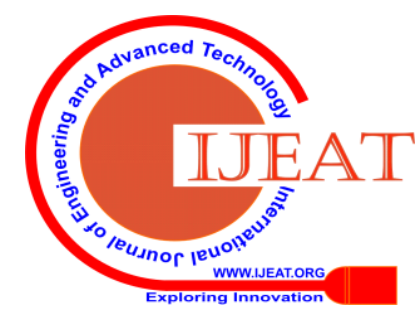




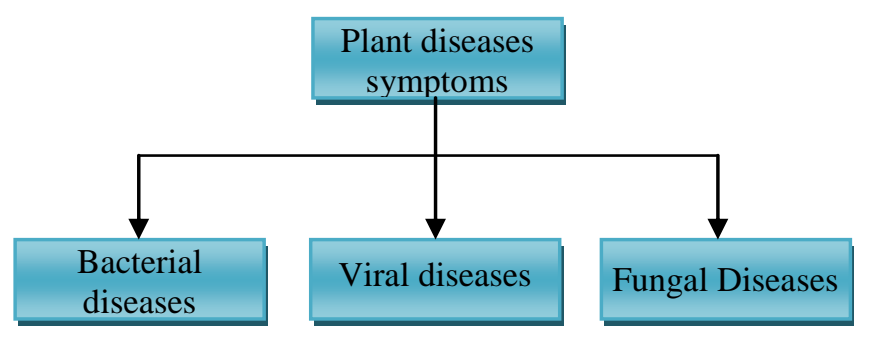

Figure 1: Classification of plant diseases

\section{A. Bacterial disease symptoms}

The disease is identified with the small spots of pale green color. These spots look like as water- soaked. The contusion on the leaf expands and it finally comes with dry and lifeless spots as delineated in figure 2(a). The discern shows the leaf with brownish black spot on the foliage. These spots can also be of the yellow color and are normally of the equal size. Also in the dry environmental conditions, the spots show up as the speckles on the leaf.

\section{B. Viral disease symptoms}

Amongst all the diseases of the plant, viruses harm the plants which are the most strenuous to diagnose. Virus in the plants produces the signs which that may be misunderstood as the deficiency of nutrients in plant or the injury caused by herbicide. Leafhoppers, cucumber beetles and whiteflies are the insects which are considered as the common carriers of the disease viz. Mosaic Virus. In this disease, the leaves are affected and different patterns with yellow or green spots are appeared on the foliage, which is shown in figure 2(b). Also this, the leaves can get wrinkly, curled and their boom would possibly be stunted.

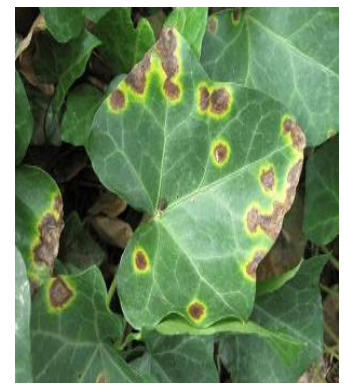

(a)Microbial Leaf Spot

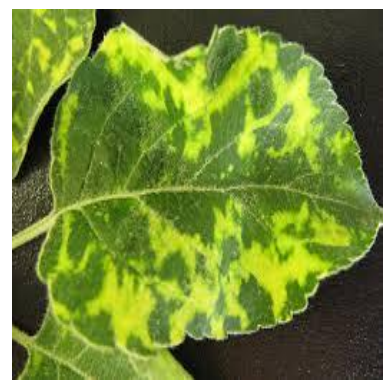

(b) Mosaic Virus
Figure 2: Microbial and Viral diseases

\section{Fungal illness symptoms}

The fungal ailment in the plants is shown in figure 3. Early blight and Late blight are the two stages of the fungal disease. Basically, Alternaria solani is the fungi that are responsible to cause early blight shown in figure 3(a).

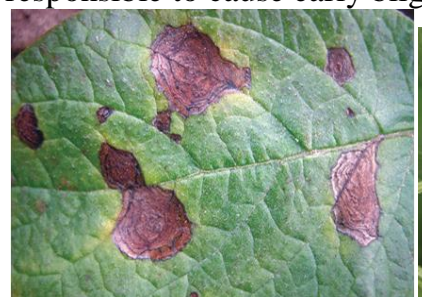

(a)Early blight

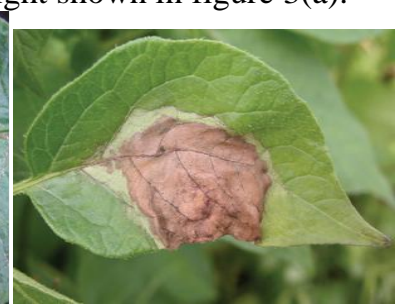

(b) Late blight
Figure 3: Fungal diseases of plants
Firstly, small spots like concentric rings are formed on the older leaves and it spreads outwards. The spots on the leaves are turned yellow when early blight is matured. Late blight is shown in figure 3(b) caused by the fungus Phytophthora infesters. It also happens to the older leaves. The spots look like water-soaked and have gray-green color. Matured late blight causes the spots to get darker with the growth of white fungi on the undersides.

\section{CNN FOR DISEASE DETECTION IN PLANTS}

CNN is Convolutional neural network which is known for its efficient performance in the field of image recognition [8] [9]. CNN is capable of getting to know the elements from the coaching data sets. [10] and [11] proves this technique splendid for detecting the illness in the foliage. The procedures designed on the basis of CNN performs their operations These purposes of CNN primarily based plant diseases diagnosis have been performed on the leaves and it has been assumed that the signs and symptoms received from one leaf shown solely one disease. In contrast, more than one ailment can appear in the discipline stipulations in accordance to the signs of present on the equal leaf. It impacts the accurateness of diagnosing plant disorder (leaf-wise) [12]. Thus, the detection of the disease in terms of symptoms wise is an important addition in this field. However, CNN has some major processes which are used to recognize the status of healthy plants. The methodology used for disease detection in the plants is portrayed into a flow chart shown in figure 3. Following are the processes which are carried out in order to detect the disease [13]:

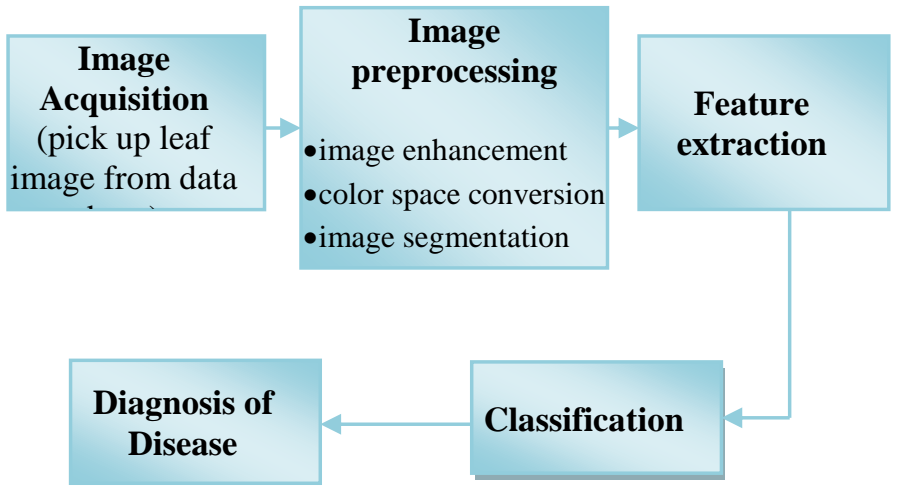

Figure 4: Flowchart of methodology

\section{A. Image acquisition}

In CNN, image acquisition is the first process. In order to achieve the better results, the image must be of high resolution i.e. pictures must be taken with the digital camera to attain better quality images. An image data base is made according to the types of the diseases in the foliage. This information base bears the accountability of offering higher efficacy of the classifiers that subsequently determines the performance of the algorithm in phrases of robustness.

\section{B. Image pre-processing}

After the above mentioned step, the next phase is the preprocessing of the image.

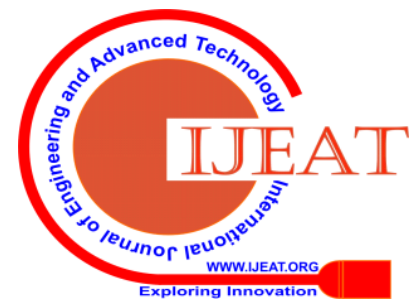


This stage is included in CNN to enhance the data of the image like undesired distortions. It also improves the features of the image so that further processing and analysis can be carried out in a facile way. This segment in addition entails exclusive techniques such as image enhancement, conversion of coloration space, and picture segmentation. RGB pictures of leaves are taken and they are changed into the shade space representation. It is carried out in order to specify the colorings in standard ordinary way. This manner can be carried out on the groundwork of the particular region, edge, and threshold and so on. [14]

\section{Feature extraction}

When the image is segmented in the pre-processing task, the affected area of the leaf is extracted. In this step, the indispensable aspects of the affected place on the leaf are extracted. Further, these aspects are used to determine the country of the given sample. In fact, the function of picture consists of the shape, colors and texture. The texture of the plant leaves is viewed as the most important function for the classification of the pants. Feature classification covered the evaluation and extraction of the elements of a texture.

\section{Classifier}

After extraction of the features, the statistics is tested and trained via the classifiers of neural network such as Radial foundation function, K-nearest neighbor, and SVM and Artificial neural networks. The classification is the final step in CNN which eventually determines the disorder induced to the plant and then plants can be recognized.

\section{LITERATURE SURVEY}

In this section, the strategies opted through a number authors are mentioned. There are exclusive applied sciences which are utilized to detect the plant diseases in order to diagnose them and enlarge the productivity in the subject of agriculture.

U. P. Singh et a. in [15] a multilayer convolutional neural community (MCNN) to perceive the mango leaves infected with Anthracnose fungal disease. The real tie contrast is carried out through taking into account both wholesome and affected leaves. The writer demonstrated better results than other methods in phrases of the accuracy.

Barbedo et al. [12] put forward a mechanism to detect the disease on the basis of color transformations, color histograms and a classification system based on pair-wise. A huge database that included the 12 different species of the leaves infected by the diseases was used to assess the anticipated technique. The deep analysis revealed the limitations and the advantages of the proposed system. The paper also presented the comparative analysis with previously proposed techniques and provides the solution of the challenges.

M. A. Khan et al in [16] implemented a novel method to detect the diseases in the apple plant. Author used hybrid model to this end. Thus, three pipeline strategies have been followed by using preprocessing, spot segmentation, and points extraction, and classification. The effects of the proposed algorithm have been efficient.

J. Ma, et al. [17] a deep convolutional neural community (DCNN) for conducting symptom-wise identification of four cucumber diseases. The pics used for evaluation were captured underneath the field conditions. Author utilized records augmentation to enlarge the statistics sets shaped from the picture segmentation. DCNN attained higher effects in terms of the disease diagnosis. The outcomes were 93.4\% accurate. Result used to be compared with conventional tactics that validated the efficacy of DCNN in terms of robustness. Kiran R. Gavhale, and U. Gawande, Gavhale [13] described various techniques of photo processing for distinct species of the plants. According the author, Support Vector Machine (SVM), Spatial Gray-level Dependence Matrices (SGDM) K-nearest neighbor (KNN), and returned propagation neural community (BPNN), are the strategies that can be used to observe the healthy and contaminated flowers with accurateness.

Anand H.Kulkarni et al. [5] used numerous photograph processing technique to detect the sickness at the early stage. In this approach, Gabor filter was once used to extract features. The ailments were categorised using ANN. The outcomes received exhibit $91 \%$ accuracy. F. Argenti, et al. [18] introduced an algorithm that performs fast computations to determine parameters of co-occurrence matrix by using utilizing supervised getting to know and most chance to classify swiftly.

Dheeb Al Bashish, et al. [19] projected a statistical classification primarily based classifier. This neural community classifier used to be able to produce the effects with 93\% accurateness and robustness. Further, Song Kai et al. [20] proposed a strategy for detecting the ailment of maze from the photos of corns. Author used YCbCr color house science for generating the segments of ailment spots and feature extraction was carried out using Co-occurrence matrix (CCM) spatial grey level layer. The classification of this strategy used to be performed through using BP neural network.

A deep CNN based method to detect the rice disease has been put forward by Y. Lu, et al, [21]. 500 images were included in the database to assess the proposed technique. CNNs were trained in order to detect common diseases of rice. The results of the anticipated work showed $95.48 \%$ accuracy which was higher than the previously proposed techniques.

It was emphasized by LeCun et al. [22] that Deep CNN have brought advanced performance in the field of image, speech, video processing. Lately, [23], [24] used deep CNN to detect foliage diseases of various. A CNN based mechanism has been proposed to diagnose the aliment in cucumber leaf. Author performs analysis and accomplished 94.9\% accuracy in differentiating amongst zucchini yellow mosaic virus, a non-diseased class and melon yellow spot virus. Sanyal et al. utilized color texture to recognize the blast and brown spot in the photographs of rice leaves [25].

S. S. Chauhan [26] Bacterial foraging optimization primarily based Radial Basis Function Neural Network (BRBFNN) to robotically recognize the plant diseases. Author has taken into account a variety of illnesses such as leaf curl, late blight, leaf spot, cedar apple rust, early blight, frequent rust. This technique greater the overall performance of the device as characteristic extraction of the frequent characteristic was once performed. The effects higher accuracy. Tallha Akram et al. [27] designed a system that can classify the ailments of foliage. The designed algorithm converts the photograph into three coloration spaces and approaches them concurrently. This algorithm worried characteristic vector construction, identification of salient areas and contrast stretching.

Published By:

Blue Eyes Intelligence Engineering 
Author also introduced the framework of primary OnChip communication. This framework made the superb connection between three digital sign processing cores and every core procedure its personal coloration space. The outcomes have been received in phrases of accuracy and computation time gadget was in a position to surpass the existing systems.

Sladojevic et al. [28] projected a method to diagnose plant ailment via using the pictures of the leaves on hand on internet. Total 13 diseases and 5 vegetation were protected to examine the proposed system. The accuracy of both systems used to be got as $91 \%$ and $98 \%$.

Pawara et al. [29] described the comparative evaluation of conventional procedures and CNN fashions in phrases of plant recognition. Author has used three databases which contained facts in shape of snap shots of vegetation and fruits, or plant leaves, and conclude that CNNs substantially surpassed the earlier proposed techniques. Eventually, Fuentes et al. [30] proposed a mannequin in CNN in order to pick out and diagnose the tomato illnesses and pests and provided higher results.

Overall, it is found from the literature survey that various strategies are opted through the researchers for figuring out the ailments of special plant species. Convolutional Neural Network is emerged as an efficient method of plant disorder detection and diagnosis. The outcomes of this method are proven in the following figure. The contrast is carried out with PSO, SVM and RBFNN techniques. It is ensured that MCNN is greater high-quality than aforementioned methods in phrases of accuracy, lacking document charge and false record fee.

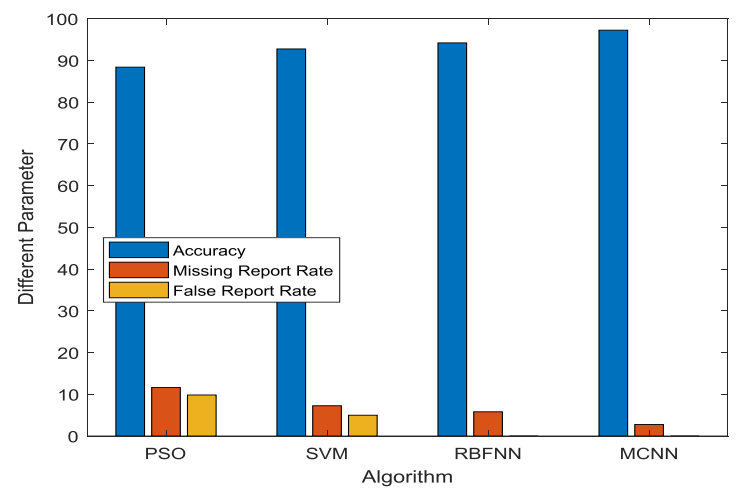

Figure 5: Comparative of different parameters.

\section{V.PROPOSED METHOD}

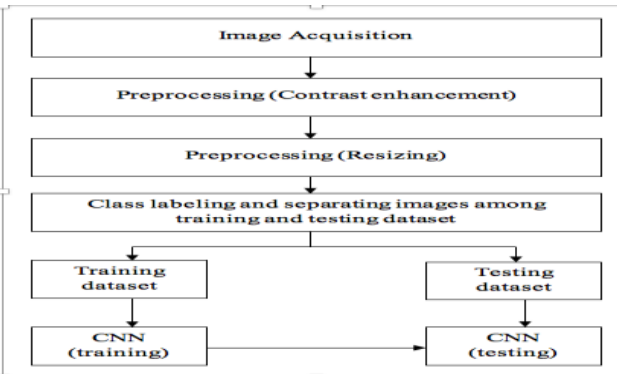

Figure 6: Flow Chart of Proposed work

In this era of research, a number of deep learning architectures have been proposed by various authors. Among these, CNN is one of the most popularly deployed deep learning models. This model possesses a complex structure as it constitutes large number of information processing layers. This multiplayer architecture differs it from the conventional Artificial Neural Networks (ANN's). They are having the capabilities of learning features from the training dataset. CNN models require very few neurons when compared with the traditional ANN but, they require a very large number of data for their training.

\section{RESULT}

Table 1 Comparison of Results for different classification methods on plant village Dataset.

\begin{tabular}{ll}
\hline Classifier & Accuracy \% \\
\hline CNN & 97 \\
\hline SVM & 94 \\
\hline
\end{tabular}

The kernel approach is described as linear kernel due to the facts factors is no longer localized. Besides, while the accuracy of train set was 0.89 , accuracy of validation set was 0.64. The accuracy Comparison for Application of Different Classification Techniques on standard datasets are calculated, Convolutional Neural Network gives the best results as depicted in table 1 .

\section{CONCLUSION}

Plant diseases are classified into three types viz, bacterial, fungal and viral. These ailments effect the growth of the plant which eventually reduces the production in agricultural industry. A significant number of techniques have been proposed to diagnose the plant malady. Some of the diseases are difficult to diagnose and some can be easily recognized. Deep neural network is used by various authors to attain the accurate results. CNN is one of the important techniques to be utilized in the felid of image processing to recognize the disease. The literature survey showed that effective techniques that assists in diagnosing the diseases accurately in their early stages. Further, it is concluded that the MCNN can be enhanced in terms of accuracy and effectiveness by introducing new methodology in CNN.

\section{REFERENCES}

1. Hill DS. Pests of crops in warmer climates and their control. Springer; 2008. ISBN 978-1-4020-6737-2, 978-1-4020-6738-9

2. Mutka, A.M., Bart, R.S., 2015. Image-based phenotyping of plant disease symptoms. Front. Plant Sci. 5, 1-8.

3. Bai, X., Li, X., Fu, Z., Lv, X., Zhang, L., 2017. A fuzzy clustering segmentation method based on neighborhood grayscale information for defining cucumber leaf spot disease images. Comput. Electron. Agric. 136, 157-165.

4. Mahlein, A.K., 2016. Plant disease detection by imaging sensorsparallels and specific demands for precision agriculture and plant phenotyping. Plant Dis. 100, 1-11.

5. Anand H. Kulkarni, Ashwin Patil R. K., Applying image processing technique to detect plant diseases, International Journal of Modern Engineering Research, vol.2, Issue.5, pp: 3661-3664, 2012.

6. LeCun, Y., Bottou, L., Bengio, Y., Haffner, P., 1998. Gradient-based learning applied to document recognition. Proc. IEEE 86 (11), 22782324

7. Dan, C., Meier, U., Masci, J., Gambardella, L.M., Schmidhuber, J. 2011. Flexible, high performance convolutional neural networks for image classification. Proceedings of the 22nd International Joint Conference on Artificial Intelligence, vol. 2, pp. 12371242.

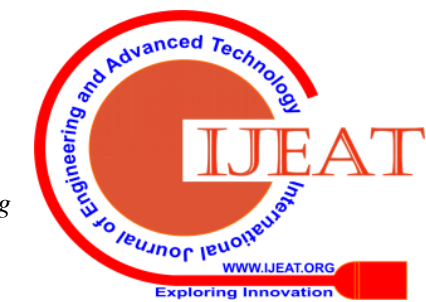


8. Grinblat, G.L., Uzal, L.C., Larese, M.G., Granitto, P.M., 2016. Deep learning for plant identification using vein morphological patterns. Comput. Electron. Agric. 127, 418-424.

9. Ghazi, M.M., Yanikoglu, B., Aptoula, E., 2017. Plant identification using deep neural networks via optimization of transfer learning parameters. Neurocomputing 235, 228-235.

10. Ferentinos, K.P., 2018. Deep learning models for plant disease detection and diagnosis. Comput. Electron. Agric. 145, 311-318

11. Mohanty, S. P., Hughes, D., Salathe, M. Inference of Plant Diseases from Leaf Images through Deep Learning. arXiv1604.03169 [cs]; 2016 1-6.

12. Barbedo, J. G. A., Koenigkan, L. V., \& Santos, T. T. (2016). Identifying multiple plant diseases using digital image processing. Biosystems Engineering, 147, 104-116.

13. Kiran R. Gavhale, and U. Gawande, "An Overview of the Research on Plant Leaves Disease detection using Image Processing Techniques," IOSR J. of Compu. Eng. (IOSRJCE), vol. 16, PP 10-16, Jan. 2014.

14. Prof.Sanjay B. Dhaygude, Mr.Nitin P. Kumbhar, Agricultural plant Leaf Disease Detection Using Image Processing, International Journal of Advanced Research in Electrical, Electronics and Instrumentation Engineering , S \& S Publication vol. 2, Issue 1, pp: 599-602, 2013

15. Uday Pratap Singh, Siddharth Singh Chauhan, Sukirty Jain, Sanjeev Jain, "Multilayer Convolution Neural Network for the Classification of Mango Leaves Infected by Anthracnose Disease" VOLUME 7, 2019, PP: 43721-43721

16. M. A. Khan et al., "An Optimized Method for Segmentation and Classification of Apple Diseases Based on Strong Correlation and Genetic Algorithm Based Feature Selection," in IEEE Access, vol. 7, pp. 46261-46277, 2019

17. J. Ma, K. Du, F. Zheng, L. Zhang, Z. Gong, and Z. Sun, "A recognition method for cucumber diseases using leaf symptom images based on deep convolutional neural network," Comput. Electron. Agricult., vol. 154, pp. 18-24, Nov. 2018.]

18. F. Argenti,L. Alparone,G. Benelli ," Fast algorithms for texture analysis using co-occurrence matrices" Radar and Signal Processing, IEE Proceedings, vol. 137, Issue 6, pp:443-448 , No. 6, December 1990]

19. Dheeb Al Bashish, M. Braik, and S. Bani-Ahmad, A Framework for Detection and Classification of Plant Leaf and Stem Diseases, 2010 International Conference on Signal and Image Processing, pp: 113118, Chennai, India, 2010.

20. Song Kai, liu zhikun, Su hang, Guo chunhong , A Research of maize disease image recognition of Corn Based on BP Networks, Third International Conference on Measuring Technology and Mechatronics Automation,pp:246-249, Shenyang, China, 2011.

21. Y. Lu, S. Yi, N. Zeng, Y. Liu, and Y. Zhang, "Identification of rice diseases using deep convolutional neural networks," NeuroComputing, vol. 267, pp. 37-384, Dec. 2017.

22. Y. LeCun, Y. Bengio, G. Hinton, Deep learning, Nature 521 (7553) (2015) 436-444.

23. R. Kawasaki, H. Uga, S. Kagiwada, H. Iyatomi, Basic study of automated diagnosis of viral plant diseases using convolutional neural networks, in: Proceedings of the International Symposium on Visual Computing (ISVC), Las Vegas, NV, USA, 2015, pp. 638-645

24. X. Zhang, Y. Lu, S. Zhang, Multi-task learning for food identification and analysis with deep convolutional neural networks, J. Comput. Sci. Technol. 31 (3) (2016) 489-500.

25. P. Sanyal, S.C. Patel, Pattern recognition method to detect two diseases in rice plants, Imaging Sci. J. 56 (6) (2013) 319-325.

26. S. S. Chouhan, A. Kaul, U. P. Singh, and S. Jain, "Bacterial foraging optimization based radial basis function neural network (RBFNN) for identification and classification of plant leaf diseases: An automatic approach towards plant pathology," IEEE Access, vol. 6, pp. 88528863, 2018.]

27. Tallha Akram et al., "Towards real-time crops surveillance for disease classification: exploiting parallelism in computer vision," Computers and Electrical Engineering, pp. 15-26, 2017, doi:10.1016/ j.compeleceng.2017.02.020.

28. Sladojevic, S., Arsenovic, M., Anderla, A., Culibrk, D., Stefanovic, D., 2016. Deep neural networks based recognition of plant diseases by leaf image classification. Computat. Intelligence Neurosci. http://dx.doi.org/10.1155/2016/3289801. Article ID: 3289801.

29. Pawara, P., Okafor, E., Surinta, O., Schomaker, L., Wiering, M. 2017. Comparing local descriptors and bags of visual words to deep convolutional neural networks for plant recognition. 6th Intl Conf. on Pattern Recognition Applications and Methods (ICPRAM 2017).
30. Fuentes, A., Yoon, S., Kim, S.C., Park, D.S., 2017. A robust deeplearning-based detector for real-time tomato plant diseases and pest recognition. Sensors 17, 2022

\section{AUTHORS PROFILE}

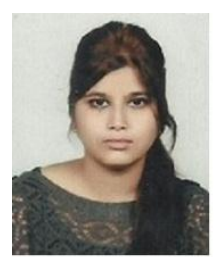

Divya Verma is a research scholar at the Computer Science Engineering Department, Chandigarh University, Punjab, India. She received her B.Tech Degree from Kurukshetra University, India. Her interests include Machine learning, Image processing, CloudComputing.

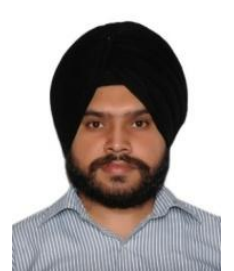

Gurpreet Singh received B.Tech. Degree in Computer Science \& Engineering from Punjab Technical University, Jalandhar in 2007 and M.Tech Degree in Computer Science \& Engineering from BBSBEC in 2011. He worked as Lecturer in the Department of Computer Science \& Engineering, IET, Bhaddal, Punjab from 2007 to 2010. He worked as Assistant Professor in the Department of Computer Science \& Engineering, IET, Bhaddal from 2010 to 2014. He was a Research Scholar in CSE Department at SLIET, Longowal from 2014 to 2018. Currently, he is working as an Assistant Professor in Chandigarh University, Punjab. His research areas include Digital Image Processing, Handwriting recognition and Natural Language Processing. He is a lifetime member of Indian Society for Technical Education.

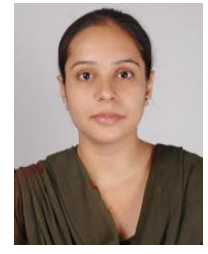

Hatesh Shyan received B.Tech Degree in Computer Science \& Engineering from Punjab Technical University in 2010 and M.Tech Degree in Computer Science \& Engineering from Punjabi University, Patiala in 2014. Currently She is working as an Assistant Professor in the Department of Computer Science \& Engineering, Chandigarh University Since 2013.Her research areas include Digital Image Processing and Internet of Things (IOT)

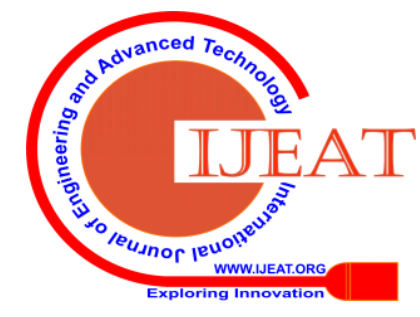

